RUNNING HEAD: Slow-paced breathing and executive function

\title{
The influence of slow-paced breathing on executive function
}

Laborde, S. ${ }^{1,2}$; Allen, M. S. ${ }^{3}$; Borges, U. ${ }^{1}$; Hosang, T. ${ }^{4}$; Furley, P. ${ }^{5}$; Mosley, E. ${ }^{6}$; Dosseville, F. ${ }^{7,8}$

${ }^{1}$ German Sport University Cologne, Institute of Psychology, Department of Performance Psychology, Germany

${ }^{2}$ Normandie Université, UFR STAPS, EA 4260 CESAMS, Caen France

${ }^{3}$ School of Psychology, University of Wollongong, Australia

${ }^{4}$ Experimental Psychology Unit, Helmut Schmidt University/University of the Federal Armed Forces, Hamburg, Germany

${ }^{5}$ German Sport University Cologne, Institute for Training and Computer Science in Sport, Germany

${ }^{6}$ Department of Sport Science and Performance, Solent University, Southampton, UK

${ }^{7}$ Normandie Université, UMR-S 1075 COMETE, Caen, France

${ }^{8}$ INSERM, UMR-S 1075 COMETE, Caen, France

Corresponding Author: Sylvain Laborde, Am Sportpark Müngersdorf, 6, Institute of Psychology, German Sport University, 50937 Cologne, Germany, s.laborde@dshskoeln.de, +4922149825701 
Abstract

The aim of this experiment was to test the immediate effects of slow-paced breathing on executive function (inhibition, working memory, and cognitive flexibility). Two theoretical models lay the groundwork for this experiment. The resonance model predicts that slow-paced breathing increases cardiac vagal activity and the neurovisceral integration model predicts that higher cardiac vagal activity leads to better executive functioning. In total, 78 participants (41 men, 37 women; $M_{\text {age }}=23.22$ years) took part in two counterbalanced experimental conditions: a 3 x 5 minute slow-paced breathing condition and a television viewing control condition. After each condition, heart-rate variability was measured and participants performed three executive function tasks: the color-word match Stroop (inhibition), the automated operation span task (working memory), and the modified card sorting task (cognitive flexibility). Results showed that performance on executive function tasks was better after slow-paced breathing compared to control, with higher scores observed for Stroop interference accuracy, automated operation span score, and perseverative errors, but not Stroop interference reaction times. This difference in executive function between experimental conditions was not mediated by cardiac vagal activity. Therefore, findings only partially align with predictions of the neurovisceral integration model and the resonance model. Slow-paced breathing appears a promising technique to improve immediate executive function performance. Further studies are recommended that address possible alternative underlying mechanisms and long-term effects. 
Slow-paced breathing and executive function

\section{The influence of slow-paced breathing on executive function}

\section{Introduction}

Improving executive functions is a constant endeavor for humans across the lifespan (Diamond \& Ling, 2016), from children (Takacs \& Kassai, 2019) to older adults (Nguyen, Murphy, \& Andrews, 2019). Of interest are both the short-term immediate effects, as well as long-term effects. Based on predictions outlined in the neurovisceral integration model (Thayer, Hansen, Saus-Rose, \& Johnsen, 2009) and the resonance model (Lehrer \& Gevirtz, 2014), the current study aimed to test the immediate effects of a relaxation technique - slow-paced breathing - on three important executive functions: inhibition, working memory, and cognitive flexibility.

According to the neurovisceral integration model (Smith, Thayer, Khalsa, \& Lane, 2017; Thayer et al., 2009), the effectiveness of the executive functioning of the prefrontal cortex can be indexed via heart rate variability (HRV), the time variation between each R peaks in the QRS complexes (Berntson et al., 1997; Laborde, Mosley, \& Thayer, 2017; Malik, 1996). More specifically, executive functioning is suggested to be indexed via the HRV parameters reflecting cardiac vagal activity - the activity of the vagus nerve regulating cardiac functioning (Thayer et al., 2009). The vagus nerve is the main nerve of the parasympathetic nervous system, and plays an essential role in selfregulation (Brodal, 2010; Thayer et al., 2009; Thayer \& Lane, 2009). Two main HRV parameters reflecting cardiac vagal activity are the root mean square of the successive differences (RMSSD) and high-frequency HRV (Berntson et al., 1997; Laborde, Mosley, et al., 2017; Malik, 1996). The relationship between cardiac vagal activity and executive functioning originates from the common structures and networks involved in cardiac and 


\section{Slow-paced breathing and executive function}

cognitive regulation (Thayer, Ahs, Fredrikson, Sollers, \& Wager, 2012). The effectiveness of executive functioning in the prefrontal cortex is supported via the optimal activation of neural networks, underlined with a flow of activity along neural pathways enabling to establish adequate mappings between input, internal states, and outputs needed to perform a given task (Miller \& Cohen, 2001), leading to flexible responses to changing environments (Thayer et al., 2009). The neurovisceral integration model assumes a linear relationship between cardiac vagal activity and executive performance. That is, a higher cardiac vagal activity will be associated with a higher executive performance.

Various methods have been proposed to increase cardiac vagal activity (Laborde, Mosley, \& Mertgen, 2018; Laborde, Mosley, \& Ueberholz, 2018), with slow-paced breathing having a strong theoretical foundation based on the resonance model (Lehrer \& Gevirtz, 2014). Slow-paced breathing is a breathing technique where the inhalation and exhalation durations are controlled ("paced"), and where breathing is performed at a slower pace (around 6 cycles per minute) than spontaneous breathing, which is usually between 12 and 20 cycles per minute in adults (Sherwood, 2006). The most common way to realize the breathing pacing is via visual stimuli (e.g., Allen \& Friedman, 2012; Laborde, Allen, Gohring, \& Dosseville, 2017; Tsai, Kuo, Lee, \& Yang, 2015), while auditive or kinesthetic methods (e.g., vibrations) have been used less frequently. The resonance model (Lehrer \& Gevirtz, 2014) explains how slow-paced breathing positively influences self-regulation mechanisms through four processes: 1) the phase relationship between heart rate (HR) oscillations and breathing at 6 cycles per minute; 2$)$ the phase relationship between $\mathrm{HR}$ and blood pressure oscillations at 6 cycles per minute; 3) the 


\section{Slow-paced breathing and executive function}

activity of the baroreflex; and 4) the resonance characteristics of the cardiovascular system. These processes strengthen homeostasis in the baroreceptor (Lehrer et al., 2006; Vaschillo, Lehrer, Rishe, \& Konstantinov, 2002; Vaschillo, Vaschillo, \& Lehrer, 2006) which results in improved gas exchanges at the level of the alveoli and increased vagal afferences (Lehrer \& Gevirtz, 2014). Both immediate (Laborde, Allen, et al., 2017; Lewis et al., 2015; Szulczewski \& Rynkiewicz, 2018; Wells, Outhred, Heathers, Quintana, \& Kemp, 2012) and long-term (Laborde, Hosang, Mosley, \& Dosseville, 2019) effects of slow-paced breathing on cardiac vagal activity have been documented in previous research. However, less is known about how slow-paced breathing might affect executive functioning.

The three core executive functions are inhibition, working memory, and cognitive flexibility (Diamond, 2013; Miyake \& Friedman, 2012; Miyake et al., 2000). Inhibition reflects being able to control attention, behavior, thoughts, and/or emotions to override a strong impulse, and to do instead what is more appropriate according to the context (Diamond, 2013). A classical test for inhibition is the color word Stroop test (Stroop, 1935), where participants are requested to read out the color in which a word is printed while ignoring the meaning of the word. In the congruent condition the color matches the meaning of the word (e.g., the word "blue" expressed in the color blue), while in the incongruent condition the color differs from the meaning of the word (e.g., the word "blue" expressed in the color red). The incongruent condition requires participants to inhibit the prepotent response of reading a word. Both the speed and accuracy of the responses can be measured. However, inhibition is primarily reflected as accuracy (error 


\section{Slow-paced breathing and executive function}

rate) (McDowd, Oseas-Kreger, \& Filion, 1995), as it captures the ability to temporarily maintain the task goal in a retrievable state (Kane \& Engle, 2003).

Previous research has found that Stroop task performance relates to cardiac vagal activity. A negative relationship has been observed between resting cardiac vagal activity and reaction times on incongruent and threat words (Johnsen et al., 2003), whereas a positive relationship has been observed between resting cardiac vagal activity and Stroop accuracy (i.e., Stroop interference score, Albinet, Abou-Dest, Andre, \& Audiffren, 2016). These two findings are in line with the neurovisceral integration model (Thayer et al., 2009). One study observed mixed-findings between resting cardiac vagal activity (assessed with high-frequency HRV) and Stroop accuracy (i.e., Stroop interference score) (Subramanya \& Telles, 2015). However, the experimental manipulation (meditation) occurring before the resting measurement might have introduced some confounding effects regarding the interpretation of high-frequency HRV, given that it is supposed to reflect cardiac vagal activity only when respiratory frequency is comprised between 9 and 24 cycles per minute (Berntson et al., 1997; Malik, 1996). As respiratory frequency was not assessed in the study, it is not possible to draw firm conclusions about cardiac vagal activity. The current experiment will investigate both accuracy and reaction time for the Stroop task controlling for respiratory frequency.

Working memory involves working with information no longer perceptually present (Baddeley \& Hitch, 1994). Stated differently, working memory involves holding information in mind and mentally working with it (Diamond, 2013). A classical test to assess working memory capacity is the automated operation span task (AOSPAN, Unsworth, Heitz, Schrock, \& Engle, 2005). The AOSPAN task requires participants to 


\section{Slow-paced breathing and executive function}

solve mathematics problems while holding a number of unrelated letters in memory.

Previous research has found a positive relationship between resting cardiac vagal activity and AOSPAN performance (Laborde, Furley, \& Schempp, 2015), and a negative relationship between task cardiac vagal activity (i.e., cardiac vagal activity measured during the AOSPAN) and AOSPAN performance when the task is realized under high pressure (Mosley, Laborde, \& Kavanagh, 2018), which might reflect the adaptation of cardiac vagal activity to the demands of the situation (Mosley et al., 2018). Positive relationships between resting cardiac vagal activity and other tasks that reflect working memory have also been reported in the literature (Hansen, Johnsen, Sollers, Stenvik, \& Thayer, 2004; Hansen, Johnsen, \& Thayer, 2003; Hansen, Johnsen, \& Thayer, 2009; Morandi et al., 2019; Pu, Schmeichel, \& Demaree, 2010; Sebastiani, Di Gruttola, Incognito, Menardo, \& Santarcangelo, 2019).

Cognitive flexibility builds on inhibition and working memory (Davidson, Amso, Anderson, \& Diamond, 2006; Diamond, 2013). Cognitive flexibility involves being able to change perspective, in particular, spatially or interpersonally (Diamond, 2013). To change perspective, there is the need to inhibit a previous perspective, and "load" a new perspective into working memory. A classical way to investigate cognitive flexibility is via the Wisconsin card sorting task (WCST, Milner, 1982; Stuss et al., 2000). In this test, each card can be sorted by color, shape, or number. Participants have to deduce the correct sorting criterion on the basis of the feedback they receive, and adapt to the new sorting rule as fast as they receive feedback that the sorting rule has changed. Previous research has shown a positive relationship between resting cardiac vagal activity and performance on the WCST (i.e., negative relationship with decision errors; Albinet, 
Slow-paced breathing and executive function

Boucard, Bouquet, \& Audiffren, 2010; Hovland et al., 2012; Mathewson, Jetha,

Goldberg, \& Schmidt, 2012), and this is similar to what has been found in other tasks of cognitive flexibility (Alba, Vila, Rey, Montoya, \& Munoz, 2019; Colzato, Jongkees, de Wit, van der Molen, \& Steenbergen, 2018).

If the influence of diverse breathing techniques on cognition has been considered in previous research (Gothe, Pontifex, Hillman, \& McAuley, 2013; Shannahoff-Khalsa, Boyle, \& Buebel, 1991; Yadav \& Mutha, 2016), the influence of slow-paced breathing on executive functioning has received little attention, with a focus on inhibition and working memory on the one hand (Prinsloo et al., 2011), and decision making on the other hand (De Couck et al., 2019). Decision making does not belong to the core executive functions but is considered a higher-order executive function that relies on core executive functions (Diamond, 2013). Prinsloo et al. (2011) used a modified Stroop test, where the inhibition component is combined to a working memory component, and participants were asked to remember how many white squares appeared on the screen. Participants were either allocated to a slow-paced breathing condition or a control condition (where they had to breathe spontaneously), for a total of 10 minutes in each conditions. The slow-paced breathing was realized with biofeedback, meaning the participants were seeing the effects of slow-paced breathing on their HRV via a dedicated device. No differences were found between conditions on the Stroop inhibition component (i.e., number of errors), but the slow-paced breathing group performed better than control on the working memory component. This important foundational research had some limitations including a small sample size ( $n=18$ for a between-subject design), an assessment of inhibition and 


\section{Slow-paced breathing and executive function}

working memory that was mixed into the same modified Stroop test, and no biomarkers of cardiac vagal activity.

In a subsequent experiment by De Couck et al. (2019), a multiple-choice test was used to investigate decision making. The test included seven questions related to a decision-making scenario in a management context. Several possible alternative answers were provided but only one answer reflected the most efficient way a manager should act.

The two conditions were a group performing slow-paced breathing for two minutes and a group breathing spontaneously during the same period (control group). The slow-paced breathing group was found to perform better on the decision-making task than the control group. This study also had some limitations with respect to the current research question, including no biomarkers of cardiac vagal activity, and the breathing was not strictly paced (participants had to mentally count how long inhalation and exhalation were lasting). Overall, research investigating the effects of slow-paced breathing on the three core executive functions is limited (with available studies characterized by some important methodological shortcomings) and has not been grounded within a solid theoretical framework.

In summary, this experiment tests predictions of the neurovisceral integration model (Thayer et al., 2009) and the resonance model (Lehrer \& Gevirtz, 2014) to investigate the immediate effects of slow-paced breathing on executive performance via an increase in cardiac vagal activity. A within-subject design was selected given the large intra-individual variability in HRV (Laborde, Mosley, et al., 2017; Quintana \& Heathers, 2014). Regarding the effects of the experimental manipulation, we hypothesized that after the experimental manipulation, in comparison to the control condition (where participants 


\section{Slow-paced breathing and executive function}

will be breathing spontaneously and watching an emotionally-neutral TV documentary), at the physiological level $\left(\mathrm{H}_{1}\right)$ in the slow-paced breathing condition participants will display a higher cardiac vagal activity (operationalized via RMSSD), a lower HR, and a lower respiratory frequency. We further hypothesized that executive performance will be higher in the slow paced breathing condition in comparison to the control condition $\left(\mathrm{H}_{2}\right)$, and that this difference will be mediated via cardiac vagal activity $\left(\mathrm{H}_{3}\right)$.

\section{Method}

\subsection{Participants}

To determine our sample size, we explored effect sizes presented in previous research of slow-paced breathing and executive functioning (De Couck et al., 2019;

Prinsloo et al., 2011). We computed an a priori power analysis using the software $G^{*}$ Power 3.0 (Faul, Erdfelder, Buchner, \& Lang, 2009). A medium effect size $(f=.25)$, for a repeated-measures MANOVA (within-factors effects), with statistical power set at .80 and an $\alpha$ level of .05 , requires a total sample size of $N=66$. We recruited a larger sample of 90 participants to allow for potential dropout or technical issues with data collection. Exclusion criteria were self-reported cardiovascular conditions, and other chronic conditions that could influence breathing or HR patterns, such as asthma, diabetes, and neurological conditions (Laborde, Mosley, et al., 2017). Because of technical issues (excessive noise or artefacts on the ECG signal) 12 participants were excluded and the final sample was comprised of $N=78$ (41 men, 37 women; $M_{\text {age }}=23.22$ years; age range $=18$-30 years; body mass index $=22.40 \pm 2.23$; waist-to-hips ratio $=$ $0.80 \pm .05)$. None of the participants were smokersing. This sample size elevated statistical power to .86 with all other parameters held constant. All participants gave 


\section{Slow-paced breathing and executive function}

written informed consent before participation, and were informed that they could withdraw from the study at any time without explanation. The experiment was conducted in line with the Declaration of Helsinki, and the protocol was approved by a human research ethics committee at the German Sport University Cologne (Project identification code 42/2015).

\subsection{Material and Measures}

\subsubsection{Cardiac vagal activity}

An ECG device was used to measure HRV (Faros $180^{\circ}$, Bittium, Kuopio,

Finland). The sampling rate was $500 \mathrm{~Hz}$. Two disposable ECG pre-gelled electrodes were used (Ambu L-00-S/25, Ambu GmbH, Bad Nauheim, Germany). The negative electrode was placed in the right infraclavicular fossa (just below the right clavicle) and the positive electrode was placed on the left side of the chest, below the pectoral muscle in the left anterior axillary line. The full ECG recording was inspected visually, and artefacts were corrected manually (Laborde, Mosley, et al., 2017). From ECG recording we extracted RMSSD using the software Kubios (University of Eastern Finland, Kuopio, Finland). RMSSD was chosen to operationalize cardiac vagal activity as it is less affected by respiration (Hill, Siebenbrock, Sollers, \& Thayer, 2009). However, given the current debate regarding whether or not to control for respiratory parameters when assessing HRV (Grossman, Karemaker, \& Wieling, 1991; Grossman \& Kollai, 1993; Laborde, Mosley, et al., 2017; Larsen, Tzeng, Sin, \& Galletly, 2010; Thayer, Loerbroks, \& Sternberg, 2011), respiratory frequency was also calculated in order to better understand whether potential changes in RMSSD are related to cardiac vagal activity or are affected by changes in respiratory frequency. Respiratory frequency was computed via the ECG 
Slow-paced breathing and executive function

derived respiration algorithm of Kubios (Tarvainen, Niskanen, Lipponen, Ranta-Aho, \&

Karjalainen, 2014).

\subsubsection{Slow-paced breathing exercise}

Similar to previous research (Laborde, Allen, et al., 2017), the slow-paced

breathing exercise was realized with the help of a video showing a ball moving up and down at the rate of six cycles per minute. The participants had to inhale continuously through the nose while the ball was going up, and exhale continuously with pursed lips when the ball was going down. This was a video capture of the software EZ-Air Plus (Biofeedback Federation of Europe $^{1}$ ). The video displayed a 3 x 5 minute slow-paced breathing exercise, with a 1-min break between each 5 minute slow-paced breathing unit, corresponding to a total of 17 minutes. The 1 minute break between each slow-paced breathing unit was introduced as some participants reported in a pilot study that 15 minutes of non-stop slow-paced breathing was very demanding. Exhalation (5.5 s) lasted slightly longer than inhalation (4.5 s) as prolonged exhalation contributes to larger beatto-beat heart fluctuations compared to a prolonged inhalation, and therefore induces a higher cardiac vagal activity (Strauss-Blasche et al., 2000). A familiarization period for slow-paced breathing was created in order for participants to become familiar with the technique. Inhaling via the nose (i.e., nasal breathing) is important because the air is warmer, cleaner and more humid (Lorig, 2011). In addition, nasal airflow was found to provoke respiratory oscillations leading to synchronized electrical activity in the piriform (olfactory) cortex, as well as in limbic-related brain areas, including amygdala and hippocampus. To sum upTherefore, inhaling throughvia the nose is suggested to provoke

\footnotetext{
${ }^{1}$ https://bfe.org/new/try-our-breath-pacer-ez-air-plus /
} 


\section{Slow-paced breathing and executive function}

228

\section{an-optimal activation of neural networks linked to stimulus processing and behavior} (Zelano et al., 2016). Exhaling takes place via the mouth, which offers less ventilatory resistance than the nasal channel (Lorig, 2011). Moreover, exhalation is realized via pursed-lips, which enables greater control over the flow of air enabling participants to match it precisely to the exhalation duration. Participants were then asked to put one hand on their chest and one hand on their stomach and were given the following instructions: "The hand on the chest should not move, only the hand on the belly should move: The belly should get bigger during the inhalation phase, and smaller during the exhalation phase." This instruction reflects an optimal activation of the diaphragm. When the diaphragm contracts and goes down, it increases the volume of the thoracic cavity and creates an area of low pressure that causes air to flow into the lungs to equalize the pressure.

During spontaneous breathing exhalation is mostly passive. However, in slowpaced breathing the forced exhalation can involve abdominal muscles which, via their contraction, help to push the diaphragm back up to the thorax, and consequently push out additional air (Lorig, 2011; West, 2015; West \& Luks, 2016). The breathing frequency is progressively decreased with 2 minute units to 10 cycles per minute, 8 cycles per minute, and then 6 cycles per minute, with a 1 minute break between each unit. The slow-paced breathing technique requires the participant to breathe in and breathe out continuously and uniformly when the ball goes up and down respectively. When this instruction is correctly followed, the sine-waves oscillation characteristics of slow-paced breathing can be observed in the R-R tachogram (Lehrer \& Gevirtz, 2014). The experimenter verified 
Slow-paced breathing and executive function

whether the participant was realizing the slow-paced breathing technique correctly during the familiarization before moving on to the next step of the experiment.

\subsubsection{TV neutral documentary (control condition)}

To serve as a control condition, a TV documentary ("Abenteuer Forschung"

[Research Adventures]) about research discoveries related to space and the universe was shown to participants for the same duration as the slow-paced breathing familiarization exercise. This TV documentary was found to be subjectively emotionally neutral in a pilot study prior to the experiment.

\subsection{Executive Function Tasks}

\subsubsection{Inhibition task}

As a measure of inhibition, we used the computerized version of the colour word match Stroop task (Stroop, 1935) with verbal responding available in the Inquisit library ${ }^{2}$, and ran it with the Inquisit software (version 5; Millisecond Software, 2017). Words appeared in 28-pt Arial font in the middle of a white screen. Three stimuli were used: colored square (congruent control stimuli), colored words displayed with the color corresponding to the word (congruent stimuli, for example the word "blue" is displayed in blue color). Colored words displayed with a color not corresponding to the word (incongruent stimuli, for example the word "blue" is displayed in red color). Participants were asked to name the color in which the word was written as fast and as accurately as possible, while ignoring the written meaning of the word. A headset (Sennheiser PC 8, Wedemark, Germany) was placed on their head for stability, with a microphone directly in front of their mouth to record the answers. The familiarization included 20 trials

${ }^{2}$ https://www.millisecond.com/download/library/stroop/ 


\section{Slow-paced breathing and executive function}

whereas for the main assessment participants completed 84 trials: 4 colors (red, green, blue, black) x 3 color stimulus congruency (congruent, incongruent, control squares) x 7 repetitions. The stimuli remained on the screen until response and latencies were measured from onset of stimuli. The inter-trial interval was $200 \mathrm{~ms}$, and the error feedback (a red cross) was $400 \mathrm{~ms}$.

\subsubsection{Working memory}

As a measure of working memory capacity, we used the AOSPAN (Unsworth et al., 2005) which is based on the original operation span task (Turner \& Engle, 1989). We used the version of the task programmed within the Inquisit database ${ }^{3}$. In this task participants are required to solve mathematics problems while trying to remember an unrelated set of letters. The task included a total of 15 trials (three trials each with $3,4,5$, 6 , and 7 letters). An example of a three item trial is $(8 / 2)-1=1$ ? (correct/incorrect?) $\rightarrow$ $\mathrm{F}$; is $(6+1)+2=8 ?($ correct/incorrect $?) \rightarrow \mathrm{P}$; is $(10+2)-5=15$ ? (correct/incorrect? $)$ $\rightarrow$ Q. After completing the three questions in this example, participants were asked to select the presented letters with a mouse click from an array of 12 potential letters in the order that they were presented (in this case F, then P, then Q). A familiarization to the task is included in the Inquisit version. The primary measure of working memory capacity is the automated operation span score (Unsworth et al., 2005), calculated as the total number of letters recalled across all error-free trials. Full task details can be found in Unsworth et al. (2005).

\subsubsection{Cognitive flexibility}

${ }^{3}$ https://www.millisecond.com/download/library/ospan/ 


\section{Slow-paced breathing and executive function}

As a measure of cognitive flexibility, we used a shorter and modified version of the Wisconsin card sorting test, the modified card sort test (Nelson, 1976). The computerized version of the Inquisit database ${ }^{4}$ was used. This test consists of two decks of 24 cards (so a total of 48) and four stimulus cards. Each card includes different colors and numbers of signs. The signs are: plus sign, star, triangle, or circle. There are one, two, three, or four signs on each card. Signs can be red, green, blue, or yellow. The participant is asked to match each new card appearing on the screen with a stimulus card. Correctly matched cards are arranged in three categories according to color, sign, and number. After the participant performs four consecutive correct matches in one category (for example, the category "color"), the computer switches without warning the rule to another category. After each choice, the participant is provided feedback about whether the response was correct or incorrect, but is not provided information regarding the correct matching category. The scoring procedures of the modified card sorting test are the same as the original Wisconsin card sorting test (Caffarra, Vezzadini, Dieci, Zonato, \& Venneri, 2004; Nelson, 1976). Participants were instructed to respond as fast and accurately as possible. Perseverative errors, which reflect the number of trials with decision errors when the matching rule has changed, was selected as the main dependent variable as it is considered the closest approximation of cognitive flexibility (Miyake et al., 2000).

\subsection{Procedure}

Participants were recruited via flyers on a campus of a single university and via social networks groups linked to the university. After contacting the experimenter team

${ }^{4}$ https://www.millisecond.com/download/library/cardsort/ 


\section{Slow-paced breathing and executive function}

who first screened for potential exclusion criteria, they received an email containing the details about the experiment. Each participant was required to take part to two testing sessions (lasting around 100 minutes each; see Figure 1). The order of the experimental conditions was counterbalanced across participants. The two sessions were separated by one week, to keep learning effects to a minimum, and took place at the same time of day, given chronotype can influence HRV (van Eekelen, Houtveen, \& Kerkhof, 2004) and cognitive performance (Folkard, 1990). Prior to the testing sessions, participants were instructed not to drink or eat anything but water during the $2 \mathrm{~h}$ before the experiment, nor to do any strenuous exercise or drink alcohol in the $24 \mathrm{~h}$ before the experiment (Laborde, Mosley, et al., 2017).

At the beginning of the experiment, participants were asked to complete an informed consent form, and at the beginning of each testing session they completed a questionnaire regarding variables potentially influencing HRV (Laborde, Mosley, et al., 2017), in order to control whether the information sent via email has been abided. The participants were then asked to turn off their smartphone. The full experiment was protocolled by the experimenter. The course of events in both conditions was identical, except for the familiarization to slow-paced breathing and the slow-paced breathing exercise in the slow-paced breathing condition, which were paralleled in the control condition with watching the neutral TV documentary. At the beginning of the slow-paced breathing condition, participants received a short introduction video on how to perform the technique correctly, and the correct execution was checked by the experimenter. All participants managed to perform correctly the slow-paced breathing technique. 


\section{Slow-paced breathing and executive function}

The experiment then continued either with the slow-paced breathing exercise, or

with watching the TV documentary. A HRV resting measure was taken before and after this step. The HRV resting measure lasted 5 minutes, based on the Task Force recommendations (Malik, 1996). The participants then performed the three executive cognitive tests: the color word Stroop test, the automated operation span, and the modified card sorting test. The order of the tests was randomized across participants.

However, for each participant the order was kept the same across experimental sessions. We used a 15 inch flat-screen monitor (Dell GmbH, Frankfurt, Germany; 1,280 x 960 pixels at $60 \mathrm{~Hz}$ ) at a viewing distance of $60 \mathrm{~cm}$ for all tests. The HRV resting measure was realized in a sitting position with eyes closed, knees at $90^{\circ}$, hands on the thighs. The same body position was kept for all cognitive tests, and the participants were asked to move as little as possible during the experiment. At the end of the second testing session, participants were debriefed and thanked. The full experimental procedure is depicted in Figure 1.

Insert Figure 1 near here

\subsection{Data Analysis}

For the HRV data, RMSSD was extracted from the Kubios output. Respiratory frequency (the number of respiratory cycles per minute) was obtained multiplying the EDR (ECG derived respiration) value obtained via the Kubios algorithm by 60 (Tarvainen et al., 2014). All participants were breathing at six cycles per minute during the slow-paced breathing technique. For inhibition, accuracy was operationalized via the number of incorrect answers retrieved for the squares, congruent stimuli, and incongruent stimuli. Regarding reaction times, only the reaction times of the correct answers was 


\section{Slow-paced breathing and executive function}

analyzed. Reaction times data were subjected to two filters. In the first filter, trials with response times lower than $200 \mathrm{~ms}$ and higher than $3000 \mathrm{~ms}$ were excluded in order to control for extreme results (see Putman \& Berling, 2011). The second filter then screened for reaction times higher or lower than two standard deviations from the mean. These were also removed (Dresler, Meriau, Heekeren, \& Van Der Meer, 2009). Working memory capacity was operationalized as the total number of letters recalled across all error-free trials. Cognitive flexibility was operationalized as the total number of perseverative errors, corresponding to a card matching error when the previous rule had changed (Nelson, 1976).

Data were checked for outliers and normality. A total of $0.90 \%$ univariate outliers cases were found and winsorized (+/- 2.58, Tabachnick \& Fidell, 2012). Multivariate outliers were checked using Mahalanobis distance, with none identified. The behavioral data related to the executive functions dependent variables were normally distributed. The physiological data (RMSSD, HR, respiratory frequency) were not normally distributed, thus a log-transformation ( $\log 10)$ was applied to achieve normal distribution, and this is consistent with previous HRV research (Laborde, Mosley, et al., 2017). For the physiological data, we ran analyses with the log-transformed values. However, for descriptive values we report the raw data for an easier interpretation.

As a manipulation check, we analyzed the difference between the resting HRV measurement before the experimental manipulation (slow-paced breathing vs. TV documentary) and after the experimental manipulation, for $\log 10 \mathrm{HR}, \log 10 \mathrm{RMSSD}$, and $\log 10$ respiratory frequency. For the experimental manipulation check, a repeatedmeasures MANOVA was conducted, with time as independent variable (pre vs. post) and 
Slow-paced breathing and executive function

with three dependent variables: $\log 10 \mathrm{HR}, \log 10 \mathrm{RMSSD}, \log 10$ respiratory frequency.

Based on our hypotheses, we focus on the condition $\mathrm{x}$ time interaction. For $\log 10$

RMSSD, the analysis is first run without covariates and then with covariates included

(session order, age, sex, body mass, waist-to-hip ratio), in order to see whether individual

Commented [SL1]: @Emma: Would you call it like this? difference factors affect results.

Regarding our main hypotheses, we conducted a 2 (time: pre vs. post) x 2

((Shannahoff-Khalsa et al., 1991)_condition: slow-paced breathing vs. control) repeatedmeasures MANOVA, with four dependent variables: 1) accuracy (error rate to incongruent stimuli) and 2) reaction times (incongruent stimuli-congruent stimuli) for the Stroop interference (operationalization of inhibition), 3) the automated operation span (operationalization of working memory), and 4) the number of perseverative errors (operationalization of cognitive flexibility). Regarding Stroop interference accuracy, account errors made with congruent stimuli were not considered in the calculation given there were none. Significant interactions were followed-up using independent samples $t$ tests with Bonferonni corrected significance levels. Where a significant effect for slowpaced breathing was found on an executive function variable, potential mediation via $\log 10$ RMSSD was tested using PROCESS statistical software (Hayes, 2013). This custom dialog tests the total, direct, and indirect effect of an independent variable on a dependent variable through a proposed mediator and allows inferences regarding indirect effects using percentile bootstrap intervals.

\section{Results}

\subsection{Slow-paced Breathing and Physiological Measures}


Slow-paced breathing and executive function

Descriptive statistics for the physiological variables concerning the manipulation

Insert Table 1 near here

A repeated-measures MANOVA was conducted and showed an overall significant interaction effect for condition $\mathrm{x}$ time, Wilks' $\lambda=.515, F(3,75)=23.6, p<$ .001 , partial $\eta^{2}=.49$. Univariate ANOVAs with Greenhouse-Geisser corrections were then ran for each physiological variable. A significant condition $\mathrm{x}$ time interaction effect was found for $\log 10 \mathrm{HR}, F(1,77)=14.9, p<.001$, partial $\eta^{2}=.16$. Four follow-up posthoc $t$-tests were conducted with Bonferroni correction $(\alpha=.0125)$. A significant difference was found in the slow-paced breathing condition, with $\log 10 \mathrm{HR}$ at Time 2 ( $M$ $=1.84, S D=0.70)$ being significantly lower than at Time $1(M=1.85, S D=0.67), t(77)$ $=4.24, p<.001, d=0.48$. No other significant differences were found for the post-hoc tests with $\log 10 \mathrm{HR}$. No significant condition $\mathrm{x}$ time interaction effect was found for $\log 10$ RMSSD $F(1,77)=3.74, p=.057$, partial $\eta^{2}=.05$. Integrating the covariates age, session order, sex, body mass, and waist-to-hip ratio did not change these results, $F(1$, 72) $=1.77, p=.215$, partial $\eta^{2}=.03$. 


\section{Slow-paced breathing and executive function}

A significant condition $\mathrm{x}$ time interaction effect for $\log 10$ respiratory frequency was found $F(1,77)=38.53, p<.001$, partial $\eta^{2}=.33$. Four follow-up post-hoc $t$-tests (with Bonferroni correction $\alpha$ at .0125) showed a significant difference between both conditions at Time 1 , with $\log 10$ respiratory frequency in the slow-paced breathing condition $(M=1.01, S D=0.71)$ being lower than in the control condition $(M=1.13, S D$ $=0.71), t(77)=12.47, p<.001, d=1.41$. A significant difference was found between both conditions at Time 2, with $\log 10$ respiratory frequency in the slow-paced breathing condition $(M=1.08, S D=0.74)$ being significantly lower than the control condition $(M=$ $1.13, S D=0.73), t(77)=5.39, p<.001, d=0.61$. A significant difference was found in the slow-paced breathing condition between Time 1 and Time 2, with $\log 10$ respiratory frequency at Time $1(M=1.01, S D=0.71)$ being significantly lower than the $\log 10$ respiratory frequency at Time $2(M=1.08, S D=0.74), t(77)=7.18, p<.001, d=0.81$.

\subsection{Slow-paced Breathing and Behavioral Measures}

Descriptive statistics for behavioral measures are reported in Table 2, and correlation matrices between all study variables are reported in the Supplementary File.

For the MANOVA, a significant overall effect for condition was found, Wilks' $\lambda$ $=.70, F(4,74)=4.69, p=.002$, partial $\eta^{2}=.20$. Univariate ANOVAs with GreenhouseGeisser corrections showed a significant effect for Stroop accuracy, $F(1,77)=9.21, p=$ .003 , partial $\eta^{2}=.11$. No significant effect was found for Stroop response time, $F(1,77)=$ $0.81, p=.372$, partial $\eta^{2}=.01$. A significant effect for working memory capacity was also found, $F(1,77)=5.66, p=.020$, partial $\eta^{2}=.07$. A significant effect for cognitive flexibility was also found, $F(177)=5.32, p=.024$, partial $\eta^{2}=.07$. 
Slow-paced breathing and executive function

Insert Table 3 near here

\subsection{Mediation Analysis}

To test whether the effect of slow-paced breathing on executive functions was mediated by RMSSD, the experimental condition, coded as slow-paced breathing (1) or control (2), was entered as the independent variable, the variables operationalizing executive functions (Stroop accuracy, automated operation span score, perseverative errors) were entered successively as dependent variables, and RMSSD was entered as mediator variable. Using a 10,000 resampling rate, the results from the bootstrapped mediation analyses revealed no significant indirect effect for Stroop accuracy (95\% CI: $.07, .05)$, no significant indirect effect for automated operation span score (95\% CI: 1.60, 2.09) and no significant indirect effect for perseverative errors (95\% CI: $-.07, .05)$.

\section{Discussion}

This experiment tested the effects of slow-paced breathing on immediate executive functioning (inhibition, working memory, cognitive flexibility). Based on the resonance model (Lehrer \& Gevirtz, 2014), we hypothesized that cardiac vagal activity operationalized via RMSSD would be higher, and HR and respiratory frequency would be lower, after the experimental manipulation with slow-paced breathing compared to control $\left(\mathrm{H}_{1}\right)$. This hypothesis was partially supported. No difference between conditions was observed for RMSSD, while respiratory frequency and HR were significantly lower in the experimental group compared to control. Based on the neurovisceral integration model (Smith et al., 2017; Thayer et al., 2009), we also hypothesized that cognitive functioning would be better in the experimental group compared to control $\left(\mathrm{H}_{2}\right)$ and that RMSSD would mediate this difference in executive functioning $\left(\mathrm{H}_{3}\right)$. These hypotheses 


\section{Slow-paced breathing and executive function}

were also partially supported. Participants did show better executive functioning after slow-paced breathing compared to control (for Stroop interference accuracy, working memory capacity, cognitive flexibility, but not Stroop interference response time), but this change in executive functioning was not mediated by RMSSD.

Regarding the physiological effects of the slow-paced breathing exercise, compared to the control condition, HR and respiratory frequency were lower, supporting our predictions, but contrary to what we hypothesized no significant difference was found for RMSSD. Based on the resonance frequency model (Lehrer \& Gevirtz, 2014), slowpaced breathing at $6 \mathrm{cpm}$ is thought to increase vagal afferences and the longer exhalation phase in comparison to the inhalation phase is supposed to activate the parasympathetic nervous system (Strauss-Blasche et al., 2000). This is based on the coupling of heart beat and respiration with the respiratory sinus arrhythmia, where inhalation is linked to faster HR, and exhalation to slower HR (Angelone \& Coulter, 1964; Yasuma \& Hayano, 2004). The null finding for RMSSD might reflect the fact that an optimal slow-paced breathing frequency, referred to as the resonance frequency (Vaschillo et al., 2006), is specific to each individual. As mentioned above, the value of 6 cycles per minute was chosen to match respiratory sinus arrhythmia to the inherent oscillations in HR linked to blood pressure modulation at $0.1 \mathrm{~Hz}$ in order for the two signals to summate and produce larger variations in HR (Lehrer \& Gevirtz, 2014). However it is possible that depending on physical characteristics such as height (Vaschillo et al., 2006) the resonance frequency differed across individuals, and that an individualized breathing frequency determined with an ad-hoc protocol (Lehrer, Vaschillo, \& Vaschillo, 2000) might have produced larger increases in cardiac vagal activity (Steffen, Austin, DeBarros, \& Brown, 2017). 


\section{Slow-paced breathing and executive function}

Two different patterns were observed for HR and respiratory frequency. HR was

found to be lower after the slow-paced breathing exercise than after watching the TV documentary, which would to some extent reflect the relaxation effect associated with slow-paced breathing (Lehrer \& Gevirtz, 2014). A different pattern was observed for respiratory frequency given that it was found to be lower in the slow-paced breathing condition in comparison to the control condition before performing the slow-paced breathing technique. This is most likely due to the fact that in the slow-paced breathing condition, participants completed a familiarization exercise that decreased their breathing frequency (see Figure 1) prior to the first resting measurement. This reduced breathing pattern remained to some extent during the first resting measurement. The respiratory frequency then increased in the slow-paced breathing condition (when comparing the measurements before and after performing the slow-paced breathing exercise), that might be due to the participants becoming habituated to the reduced respiratory frequency while performing the technique, and returning faster to their normal respiratory frequency.

However the fact that respiratory frequency was still lower in the slow-paced breathing condition than in the control condition (after the slow-paced breathing exercise and before starting the executive functioning tasks) supports the predicted effects of the slowpaced breathing technique at the respiratory level (Lehrer \& Gevirtz, 2014).

Regarding our second hypothesis, executive functioning improved after the slowpaced breathing exercise in comparison to the control condition, for 1) inhibition with less errors for the incongruent stimuli (i.e., better Stroop interference accuracy), for 2) working memory capacity with a higher automated operation span score, and for 3) cognitive flexibility with less perseverative errors. No change was found on Stroop 
Slow-paced breathing and executive function

interference reaction time. Nevertheless, regarding hypothesis 3 , the results are not in line with predictions of the neurovisceral integration model (Smith et al., 2017; Thayer et al., 2009), given the improvement in executive functioning was not mediated by RMSSD.

\section{However, it is still noteworthy to remarkimportant to note that in both the control and in} the slow-paced breathing condition, resting RMSSD before the executive tasks was correlated positively with performance on the Stroop interference accuracy and on the automated operation span score (while no associations were found with the Stroop interference reaction times and perseverative errors on the WCST), which would be partially in line with the neurovisceral integration model (Smith et al., 2017; Thayer et al., 2009). Taken together, these findings could suggest that other mechanisms might be contributing to the effects of slow-paced breathing on executive functioning. This might include improved functional connectivity in brain networks (Mather \& Thayer, 2018). To explain, the slow oscillations in HR produced by slow-paced breathing are suggested to have the potential to strengthen brain network dynamics, especially in medial prefrontal regulatory regions that are particularly sensitive to physiological oscillations (Mather \& Thayer, 2018), and which are responsible for cognitive control and emotion regulation (Thayer et al., 2012). In order to uncover the mechanisms at work, that might be involved in oxygenation, blood flow or electophysiological signals in brain areas associated with executive functions, future research should investigate the effects of slow-paced breathing using brain imaging techniques such as EEG, $f$ NIRS, or $f$ MRI.

Our experiment has some notable limitations that need to be considered when interpreting main findings. First, our control condition involved spontaneous breathing while the participants were watching a neutral TV documentary. Therefore, we cannot 
Slow-paced breathing and executive function

rule out the possibility that participants in the control group were actively focusing on their breathing, and future studies should include a control condition that includes a similar focus on breathing at a different pace, perhaps 12 cycles per minute (Tsai et al., 2015) that would match typical lower range spontaneous respiratory frequencies (Sherwood, 2006). Second, respiratory frequency was obtained via a dedicated algorithm from Kubios (Tarvainen et al., 2014). However, a more precise assessment of respiratory frequency such as a respiratory belt or a pneumotachograph (Egizio, Eddy, Robinson, \& Jennings, 2011; Quintana \& Heathers, 2014) and the assessment of other respiratory related variables (e.g., respiratory depth, gas exchanges) could prove helpful in explaining the effects of slow-paced breathing on executive functioning (Lorig, 2011; Ritz et al., 2002). Third, the initial physical activity level of the participants may have an influence on the results, and future research should consider assessing it using a standardized questionnaire such as the International Physical Activity Questionnaire (Craig et al., 2003). Fourth, some meaningful variations could be introduced to the slowpaced breathing technique practiced in this study. For example, having a break introduced between inhalation and exhalation, or between exhalation and inhalation, could have different physiological consequences (Reyes del Paso, Munoz Ladron de Guevara, \& Montoro, 2015; Skow, Day, Fuller, Bruce, \& Steinback, 2015). In our case, future studies should investigate the use of a post-exhalation break, given that it appears to increase cardiac vagal activity more than no break (Russell, Scott, Boggero, \& Carlson, 2017).

\section{Conclusion}

In conclusion, this experiment tested the effects of slow-paced breathing on immediate executive functioning in a sample of young adults. The slow-paced breathing 
Slow-paced breathing and executive function

exercise was able to decrease HR and respiratory frequency, while no change was observed for RMSSD. An increase in executive functioning was observed for inhibition, working memory, and cognitive flexibility, after the slow-paced breathing exercise compared to control. However, this increase in executive performance was not mediated by RMSSD. Therefore, the influence of slow-paced breathing on executive functioning cannot be explained by an increase in cardiac vagal activity as predicted by the neurovisceral integration model (Smith et al., 2017; Thayer et al., 2009). Further research might want to test (using brain imaging) whether brain network dynamics are involved in the association between slow-paced breathing and executive functioning. Finally, at the applied level, these findings may have implications for individuals looking for a quick fix and easy method forto alter their executive functions, for example to better execute cognitively demanding tasks in their jobs. 
Slow-paced breathing and executive function

\section{References}

Alba, G., Vila, J., Rey, B., Montoya, P., \& Munoz, M. A. (2019). The Relationship Between Heart Rate Variability and Electroencephalography Functional Connectivity Variability Is Associated With Cognitive Flexibility. Frontiers in Human Neuroscience, 13, 64. doi:10.3389/fnhum.2019.00064

Albinet, C. T., Abou-Dest, A., Andre, N., \& Audiffren, M. (2016). Executive functions improvement following a 5-month aquaerobics program in older adults: Role of cardiac vagal control in inhibition performance. Biological Psychology. doi:10.1016/j.biopsycho.2016.01.010

Albinet, C. T., Boucard, G., Bouquet, C. A., \& Audiffren, M. (2010). Increased heart rate variability and executive performance after aerobic training in the elderly. European Journal of Applied Physiology, 109, 617-624. doi:10.1007/s00421-010-1393-y

Allen, B., \& Friedman, B. H. (2012). Positive emotion reduces dyspnea during slow paced breathing. Psychophysiology, 49(5), 690-696. doi:10.1111/j.1469-8986.2011.01344.x

Angelone, A., \& Coulter, N. A., Jr. (1964). Respiratory Sinus Arrhythemia: A Frequency Dependent Phenomenon. Journal of Applied Physiology, 19, 479-482. doi:10.1152/jappl.1964.19.3.479

Baddeley, A. D., \& Hitch, G. J. (1994). Developments in the concept of working memory. Neuropsychology, 8(4), 485-493. doi:10.1037/0894-4105.8.4.485

Berntson, G. G., Bigger, J. T., Eckberg, D. L., Grossman, P., Kaufmann, P. G., Malik, M., . . . van der Molen, M. W. (1997). Heart rate variability: origins, methods, and interpretive caveats. Psychophysiology, 34, 623-648.

Brodal, P. (2010). The central nervous system - Structure and function. New York: Oxford University Press.

Caffarra, P., Vezzadini, G., Dieci, F., Zonato, F., \& Venneri, A. (2004). Modified Card Sorting Test: normative data. Journal of Clinical and Experimental Neuropsychology, 26(2), 246250. doi:10.1076/jcen.26.2.246.28087

Colzato, L. S., Jongkees, B. J., de Wit, M., van der Molen, M. J. W., \& Steenbergen, L. (2018) Variable heart rate and a flexible mind: Higher resting-state heart rate variability predicts better task-switching. Cognitive, Affective, \& Behavioral Neuroscience, 18, 730-738. doi:10.3758/s13415-018-0600-x

Craig, C. L., Marshall, A. L., Sjostrom, M., Bauman, A. E., Booth, M. L., Ainsworth, B. E., .. . Oja, P. (2003). International physical activity questionnaire: 12-country reliability and validity. Medicine \& Science in Sports \& Exercise, 35(8), 1381-1395. doi:10.1249/01.MSS.0000078924.61453.FB

Davidson, M. C., Amso, D., Anderson, L. C., \& Diamond, A. (2006). Development of cognitive control and executive functions from 4 to 13 years: evidence from manipulations of memory, inhibition, and task switching. Neuropsychologia, 44(11), 2037-2078. doi:10.1016/j.neuropsychologia.2006.02.006

De Couck, M., Caers, R., Musch, L., Fliegauf, J., Giangreco, A., \& Gidron, Y. (2019). How breathing can help you make better decisions: Two studies on the effects of breathing patterns on heart rate variability and decision-making in business cases. International Journal of Psychophysiology, 139, 1-9. doi:10.1016/j.ijpsycho.2019.02.011

Diamond, A. (2013). Executive functions. Annual Review of Psychology, 64, 135-168. doi:10.1146/annurev-psych-113011-143750

Diamond, A., \& Ling, D. S. (2016). Conclusions about interventions, programs, and approaches for improving executive functions that appear justified and those that, despite much hype, do not. Developmental Cognitive Neuroscience, 18, 34-48. doi:10.1016/j.den.2015.11.005 
Dresler, T., Meriau, K., Heekeren, H. R., \& Van Der Meer, E. (2009). Emotional Stroop task : effect of word arousal and subject anxiety on emotional interference. Psychological Research, 73(3), 364-371.

Egizio, V. B., Eddy, M., Robinson, M., \& Jennings, J. R. (2011). Efficient and cost-effective estimation of the influence of respiratory variables on respiratory sinus arrhythmia. Psychophysiology, 48(4), 488-494. doi:10.1111/j.1469-8986.2010.01086.x

Faul, F., Erdfelder, E., Buchner, A., \& Lang, A. G. (2009). Statistical power analyses using G*Power 3.1: tests for correlation and regression analyses. Behavior Research Methods, 41, 1149-1160. doi:10.3758/BRM.41.4.1149

Folkard, S. (1990). Circadian performance rhythms: some practical and theoretical implications. Philosophical Transactions of the Royal Society B: Biological Sciences, 327(1241), 543553.

Gothe, N., Pontifex, M. B., Hillman, C., \& McAuley, E. (2013). The acute effects of yoga on executive function. $J$ Phys Act Health, 10(4), 488-495.

Grossman, P., Karemaker, J., \& Wieling, W. (1991). Prediction of tonic parasympathetic cardiac control using respiratory sinus arrhythmia: the need for respiratory control. Psychophysiology, 28(2), 201-216.

Grossman, P., \& Kollai, M. (1993). Respiratory sinus arrhythmia, cardiac vagal tone, and respiration: within- and between-individual relations. Psychophysiology, 30(5), 486-495.

Hansen, A. L., Johnsen, B. H., Sollers, J. J., Stenvik, K., \& Thayer, J. F. (2004). Heart rate variability and its relation to prefrontal cognitive function: the effects of training and detraining. European Journal of Applied Physiology, 93, 263-272. doi:10.1007/s00421004-1208-0

Hansen, A. L., Johnsen, B. H., \& Thayer, J. F. (2003). Vagal influence on working memory and attention. International Journal of Psychophysiology, 48, 263-274. doi:10.1016/S01678760(03)00073-4

Hansen, A. L., Johnsen, B. H., \& Thayer, J. F. (2009). Relationship between heart rate variability and cognitive function during threat of shock. Anxiety, Stress, and Coping, 22, 77-89. doi:10.1080/10615800802272251

Hayes, A. F. (2013). Introduction to mediation, moderation, and conditional process analysis. New York: The Guilford Press.

Hill, L. K., Siebenbrock, A., Sollers, J. J., \& Thayer, J. F. (2009). Are all measures created equal? Heart rate variability and respiration. Biomedical sciences instrumentation, 45, 71-76.

Hovland, A., Pallesen, S., Hammar, A., Hansen, A. L., Thayer, J. F., Tarvainen, M. P., \& Nordhus, I. H. (2012). The relationships among heart rate variability, executive functions, and clinical variables in patients with panic disorder. International Journal of Psychophysiology, 86, 269-275.

Johnsen, B. H., Thayer, J. F., Laberg, J. C., Wormnes, B., Raadal, M., Skaret, E., . . Berg, E. (2003). Attentional and physiological characteristics of patients with dental anxiety. Journal of Anxiety Disorders, 17(1), 75-87.

Kane, M. J., \& Engle, R. W. (2003). Working-memory capacity and the control of attention: The contributions of goal neglect, response competition, and task set to Stroop interference. Journal of Experimental Psychology: General, 132(1), 47-70. doi:10.1037/00963445.132.1.47

Laborde, S., Allen, M. S., Gohring, N., \& Dosseville, F. (2017). The effect of slow-paced breathing on stress management in adolescents with intellectual disability. Journal of Intellectual Disability Research, 61(6), 560-567. doi:10.1111/jir.12350

Laborde, S., Furley, P., \& Schempp, C. (2015). The relationship between working memory, reinvestment, and heart rate variability. Physiology \& Behavior, 139, 430-436. doi:10.1016/j.physbeh.2014.11.036 
Laborde, S., Hosang, T., Mosley, E., \& Dosseville, F. (2019). Influence of a 30-day slow paced breathing intervention compared to social media use on subjective sleep quality and cardiac vagal activity. Journal of Clinical Medicine, 8. doi:10.3390/jcm8020193

Laborde, S., Mosley, E., \& Mertgen, A. (2018). A unifying conceptual framework of factors associated to cardiac vagal control. Heliyon, 4(12), e01002. doi:10.1016/j.heliyon.2018.e01002

Laborde, S., Mosley, E., \& Thayer, J. F. (2017). Heart Rate Variability and Cardiac Vagal Tone in Psychophysiological Research - Recommendations for Experiment Planning, Data Analysis, and Data Reporting. Frontiers in Physiology, 8, 213. doi:10.3389/fpsyg.2017.00213

Laborde, S., Mosley, E., \& Ueberholz, L. (2018). Enhancing cardiac vagal activity: Factors of interest for sport psychology. Progress in Brain Research, 240, 71-92. doi:10.1016/bs.pbr.2018.09.002

Larsen, P. D., Tzeng, Y. C., Sin, P. Y., \& Galletly, D. C. (2010). Respiratory sinus arrhythmia in conscious humans during spontaneous respiration. Respiratory Physiology \& Neurobiology, 174(1-2), 111-118. doi:10.1016/j.resp.2010.04.021

Lehrer, P. M., \& Gevirtz, R. (2014). Heart rate variability biofeedback: how and why does it work? Frontiers in Psychology, 5. doi:10.3389/fpsyg.2014.00756

Lehrer, P. M., Vaschillo, E., Lu, S. E., Eckberg, D., Vaschillo, B., Scardella, A., \& Habib, R. (2006). Heart rate variability biofeedback: effects of age on heart rate variability, baroreflex gain, and asthma. Chest, 129(2), 278-284. doi:10.1378/chest.129.2.278

Lehrer, P. M., Vaschillo, E., \& Vaschillo, B. (2000). Resonant frequency biofeedback training to increase cardiac variability: rationale and manual for training. Applied Psychophysiology \& Biofeedback, 25(3), 177-191.

Lewis, G. F., Hourani, L., Tueller, S., Kizakevich, P., Bryant, S., Weimer, B., \& Strange, L. (2015). Relaxation training assisted by heart rate variability biofeedback: Implication for a military predeployment stress inoculation protocol. Psychophysiology, 52(9), 11671174. doi:10.1111/psyp. 12455

Lorig, T. (2011). The respiratory system. In J. T. Cacioppo, L. G. Tassinary, \& G. G. Berntson (Eds.), The Handbook of Psychophysiology (pp. 231-244). New York: Cambridge University Press.

Malik, M. (1996). Heart rate variability. Standards of measurement, physiological interpretation, and clinical use. Task Force of the European Society of Cardiology and the North American Society of Pacing and Electrophysiology. European Heart Journal, 17, 354381.

Mather, M., \& Thayer, J. F. (2018). How heart rate variability affects emotion regulation brain networks. Current Opinion in Behavioral Sciences, 19, 98-104. doi:10.1016/j.cobeha.2017.12.017

Mathewson, K. J., Jetha, M. K., Goldberg, J. O., \& Schmidt, L. A. (2012). Autonomic regulation predicts performance on Wisconsin Card Sorting Test (WCST) in adults with schizophrenia. Biological Psychology, 91, 389-399. doi:10.1016/j.biopsycho.2012.09.002

McDowd, J. M., Oseas-Kreger, D. M., \& Filion, D. L. (1995). Inhibitory processes in cognition and aging. In D. F. N. \& B. C. J. (Eds.), Interference and Inhibition in Cognition (pp. 363-400). San Diego, CA: Academic Press.

Miller, E. K., \& Cohen, J. D. (2001). An integrative theory of prefrontal cortex function. Annual Review of Neuroscience, 24, 167-202.

Milner, B. (1982). Some cognitive effects of frontal-lobe lesions in man. Philosophical Transactions of the Royal Society B: Biological Sciences, 298(1089), 211-226. doi:10.1098/rstb.1982.0083 
Miyake, A., \& Friedman, N. P. (2012). The Nature and Organization of Individual Differences in Executive Functions: Four General Conclusions. Current Directions in Psychological Science, 21(1), 8-14. doi:10.1177/0963721411429458

Miyake, A., Friedman, N. P., Emerson, M. J., Witzki, A. H., Howerter, A., \& Wager, T. D. (2000). The unity and diversity of executive functions and their contributions to complex "Frontal Lobe" tasks: a latent variable analysis. Cognitive Psychology, 41(1), 49-100. doi:10.1006/cogp.1999.0734

Morandi, G. N., Lin, S. H., Lin, C. W., Yeh, T. L., Chu, C. L., Lee, I. H., . . Yang, Y. K. (2019). Heart Rate Variability is Associated with Memory in Females. Applied Psychophysiology \& Biofeedback, 44(2), 117-122. doi:10.1007/s10484-018-9425-1

Mosley, E., Laborde, S., \& Kavanagh, E. (2018). Coping related variables, cardiac vagal activity and working memory performance under pressure. Acta Psychologica, 191, 179-189. doi:10.1016/j.actpsy.2018.09.007

Nelson, H. E. (1976). A modified card sorting test sensitive to frontal lobe defects. Cortex, 12(4), 313-324.

Nguyen, L., Murphy, K., \& Andrews, G. (2019). Immediate and long-term efficacy of executive functions cognitive training in older adults: A systematic review and meta-analysis. Psychological Bulletin, 145(7), 698-733. doi:10.1037/bul0000196

Prinsloo, G. E., Rauch, H. G., Lambert, M. I., Muench, F., Noakes, T. D., \& Derman, W. E. (2011). The effect of short duration heart rate variability (HRV) biofeedback on cognitive performance during laboratory induced cognitive stress. Applied Cognitive Psychology, 25, 792-801. doi:10.1002/acp.1750

Pu, J., Schmeichel, B. J., \& Demaree, H. A. (2010). Cardiac vagal control predicts spontaneous regulation of negative emotional expression and subsequent cognitive performance. Biological Psychology, 84, 531-540. doi:10.1016/j.biopsycho.2009.07.006

Putman, P., \& Berling, S. (2011). Cortisol acutely reduces selective attention for erotic words in healthy young men. Psychoneuroendocrinology, 36(9), 1407-1417. doi:10.1016/j.psyneuen.2011.03.015

Quintana, D. S., \& Heathers, J. A. (2014). Considerations in the assessment of heart rate variability in biobehavioral research. Frontiers in Physiology, 5, 805. doi:10.3389/fpsyg.2014.00805

Reyes del Paso, G. A., Munoz Ladron de Guevara, C., \& Montoro, C. I. (2015). Breath-Holding During Exhalation as a Simple Manipulation to Reduce Pain Perception. Pain Medicine, 16(9), 1835-1841. doi:10.1111/pme.12764

Ritz, T., Dahme, B., Dubois, A. B., Folgering, H., Fritz, G. K., Harver, A., . . Van de Woestijne, K. P. (2002). Guidelines for mechanical lung function measurements in psychophysiology. Psychophysiology, 39(5), 546-567. doi:10.1017.S0048577202010715

Russell, M. E. B., Scott, A. B., Boggero, I. A., \& Carlson, C. R. (2017). Inclusion of a rest period in diaphragmatic breathing increases high frequency heart rate variability: Implications for behavioral therapy. Psychophysiology, 54(3), 358-365. doi:10.1111/psyp.12791

Sebastiani, L., Di Gruttola, F., Incognito, O., Menardo, E., \& Santarcangelo, E. L. (2019). The higher the basal vagal tone the better the motor imagery ability. Archives Italiennes De Biologie, 157(1), 3-14. doi:10.12871/00039829201911

Shannahoff-Khalsa, D. S., Boyle, M. R., \& Buebel, M. E. (1991). The effects of unilateral forced nostril breathing on cognition. Int J Neurosci, 57(3-4), 239-249. doi:10.3109/00207459109150697

Sherwood, L. (2006). Fundamentals of physiology: A human perspective (3rd ed.). Belmont, CA: Brooks/Cole.

Skow, R. J., Day, T. A., Fuller, J. E., Bruce, C. D., \& Steinback, C. D. (2015). The ins and outs of breath holding: simple demonstrations of complex respiratory physiology. Advances in Physiology Education, 39(3), 223-231. doi:10.1152/advan.00030.2015 
Smith, R., Thayer, J. F., Khalsa, S. S., \& Lane, R. D. (2017). The hierarchical basis of neurovisceral integration. Neuroscience \& Biobehavioral Reviews, 75, 274-296. doi:10.1016/j.neubiorev.2017.02.003

Steffen, P. R., Austin, T., DeBarros, A., \& Brown, T. (2017). The Impact of Resonance Frequency Breathing on Measures of Heart Rate Variability, Blood Pressure, and Mood. Frontiers in Public Health, 5, 6. doi:10.3389/fpubh.2017.00222

Strauss-Blasche, G., Moser, M., Voica, M., McLeod, D. R., Klammer, N., \& Marktl, W. (2000). Relative timing of inspiration and expiration affects respiratory sinus arrhythmia. Clinical and Experimental Pharmacology and Physiology, 27(8), 601-606.

Stroop, J. R. (1935). Studies of interference in serial verbal reactions. Journal of Experimental Psychology, 18(6), 643. doi:10.1037/h0054651

Stuss, D. T., Levine, B., Alexander, M. P., Hong, J., Palumbo, C., Hamer, L., . . Izukawa, D. (2000). Wisconsin Card Sorting Test performance in patients with focal frontal and posterior brain damage: effects of lesion location and test structure on separable cognitive processes. Neuropsychologia, 38(4), 388-402.

Subramanya, P., \& Telles, S. (2015). Performance in the Stroop Task and Simultaneously Recorded Heart Rate Variability before and after Meditation, Supine Rest and NoIntervention. International Journal of Brain and Cognitive Sciences, 4, 8-14.

Szulczewski, M. T., \& Rynkiewicz, A. (2018). The effects of breathing at a frequency of $0.1 \mathrm{~Hz}$ on affective state, the cardiovascular system, and adequacy of ventilation. Psychophysiology, 55(12), e13221. doi:10.1111/psyp.13221

Tabachnick, B., \& Fidell, L. (2012). Using multivariate statistics (6th ed.). New Jersey: Pearson.

Takacs, Z. K., \& Kassai, R. (2019). The efficacy of different interventions to foster children's executive function skills: A series of meta-analyses. Psychological Bulletin, 145(7), 653697. doi: $10.1037 /$ bul0000195

Tarvainen, M. P., Niskanen, J. P., Lipponen, J. A., Ranta-Aho, P. O., \& Karjalainen, P. A. (2014). Kubios HRV--heart rate variability analysis software. Computer Methods Programs Biomedical, 113(1), 210-220. doi:10.1016/j.cmpb.2013.07.024

Thayer, J. F., Ahs, F., Fredrikson, M., Sollers, J. J., \& Wager, T. D. (2012). A meta-analysis of heart rate variability and neuroimaging studies: implications for heart rate variability as a marker of stress and health. Neuroscience \& Biobehavioral Reviews, 36, 747-756. doi:10.1016/j.neubiorev.2011.11.009

Thayer, J. F., Hansen, A. L., Saus-Rose, E., \& Johnsen, B. H. (2009). Heart rate variability, prefrontal neural function, and cognitive performance: the neurovisceral integration perspective on self-regulation, adaptation, and health. Annals of Behavioral Medicine, 37, 141-153. doi:10.1007/s12160-009-9101-z

Thayer, J. F., \& Lane, R. D. (2009). Claude Bernard and the heart-brain connection: further elaboration of a model of neurovisceral integration. Neuroscience \& Biobehavioral Reviews, 33, 81-88. doi:10.1016/j.neubiorev.2008.08.004

Thayer, J. F., Loerbroks, A., \& Sternberg, E. M. (2011). Inflammation and cardiorespiratory control: the role of the vagus nerve. Respiratory Physiology \& Neurobiology, 178, 387 394. doi:10.1016/j.resp.2011.05.016

Tsai, H. J., Kuo, T. B., Lee, G. S., \& Yang, C. C. (2015). Efficacy of paced breathing for insomnia: enhances vagal activity and improves sleep quality. Psychophysiology, 52(3), 388-396. doi:10.1111/psyp.12333

Turner, M. L., \& Engle, R. W. (1989). Is working memory capacity task dependent? Journal of Memory \& Language, 28, 127-154.

Unsworth, N., Heitz, R. P., Schrock, J. C., \& Engle, R. W. (2005). An automated version of the operation span task. Behavior Research Methods, 37, 498-505. 
van Eekelen, A. P., Houtveen, J. H., \& Kerkhof, G. A. (2004). Circadian variation in cardiac autonomic activity: reactivity measurements to different types of stressors. Chronobiology International, 21, 107-129.

Vaschillo, E. G., Lehrer, P., Rishe, N., \& Konstantinov, M. (2002). Heart rate variability biofeedback as a method for assessing baroreflex function: a preliminary study of resonance in the cardiovascular system. Applied Psychophysiology \& Biofeedback, 27(1), $1-27$.

Vaschillo, E. G., Vaschillo, B., \& Lehrer, P. M. (2006). Characteristics of resonance in heart rate variability stimulated by biofeedback. Applied Psychophysiology \& Biofeedback, 31(2), 129-142. doi:10.1007/s10484-006-9009-3

Wells, R., Outhred, T., Heathers, J. A., Quintana, D. S., \& Kemp, A. H. (2012). Matter over mind: a randomised-controlled trial of single-session biofeedback training on performance anxiety and heart rate variability in musicians. PLOS ONE, 7, e46597. doi:10.1371/journal.pone.0046597

West, J. B. (2015). Essays on the history of respiratory physiology. New York, USA: Springer.

West, J. B., \& Luks, A. M. (2016). West's Respiratory Physiology - The Essentials. Philadelphia, USA: Wolters Kluwer.

Yadav, G., \& Mutha, P. K. (2016). Deep Breathing Practice Facilitates Retention of Newly Learned Motor Skills. Sci Rep, 6, 37069. doi:10.1038/srep37069

Yasuma, F., \& Hayano, J. (2004). Respiratory sinus arrhythmia: Why does the heartbeat synchronize with respiratory rhythm? Chest, 125, 683-690. doi:10.1378/chest.125.2.683

Zelano, C., Jiang, H., Zhou, G., Arora, N., Schuele, S., Rosenow, J., \& Gottfried, J. A. (2016).

Nasal Respiration Entrains Human Limbic Oscillations and Modulates Cognitive Function. Journal of Neuroscience, 36(49), 12448-12467. doi:10.1523/jneurosci.258616.2016 
846
Figure 1

\section{Illustration of the experimental protocol}
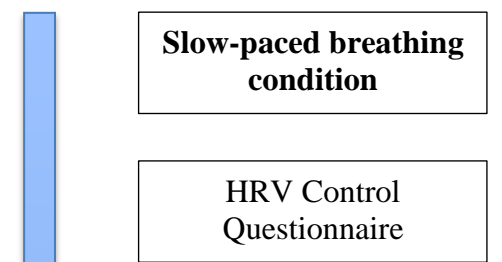

$5 \min$

ECG device
attachement

Slow-paced breathing Familiarization

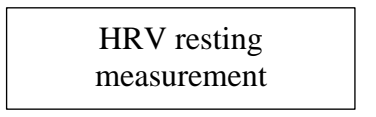

Slow-paced breathing
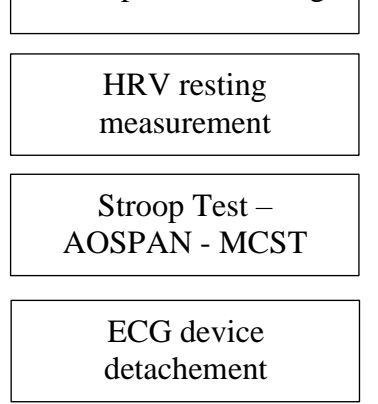

$11 \mathrm{~min}$
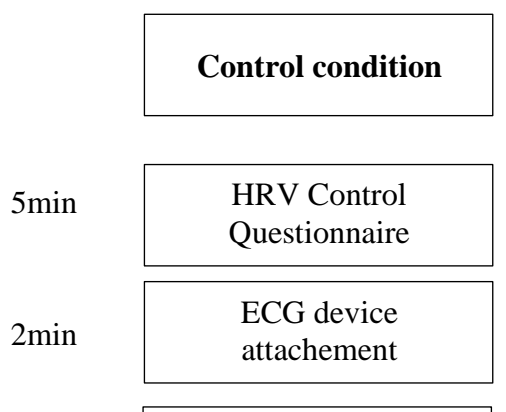

Neutral TV documentary (Part 1)
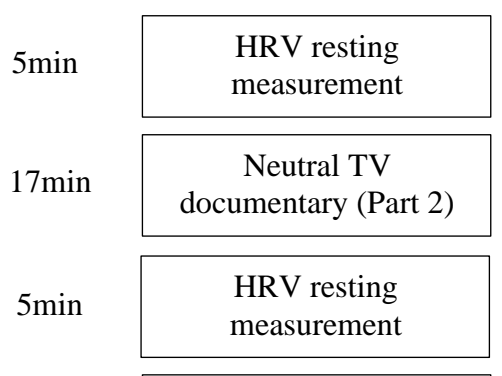

$30 \min$

Stroop Test AOSPAN - MCST

$2 \min$

\section{ECG device} detachement

Notes. HRV: heart rate variability; AOSPAN: Automated Operation Span; MCST: Modified Card Sorting

Test; ECG: electrocardiography 
Slow-paced breathing and executive function

868

Table 1

Descriptive statistics for physiological variables

\begin{tabular}{|c|c|c|c|c|c|}
\hline & & \multicolumn{2}{|c|}{$\begin{array}{c}\text { Resting measurement before } \\
\text { slow-paced breathing exercise/TV } \\
\text { documentary }\end{array}$} & \multicolumn{2}{|c|}{$\begin{array}{c}\text { Resting measurement after slow- } \\
\text { paced breathing exercise/TV } \\
\text { documentary }\end{array}$} \\
\hline & & $M$ & $S D$ & $M$ & $S D$ \\
\hline \multirow{3}{*}{$\begin{array}{l}\text { Slow-paced } \\
\text { breathing } \\
\text { condition }\end{array}$} & RMSSD & 47.97 & 28.66 & 53.43 & 35.46 \\
\hline & Heart Rate & 71.00 & 10.93 & 69.57 & 11.02 \\
\hline & $\begin{array}{l}\text { Respiratory } \\
\text { Frequency }\end{array}$ & 10.48 & 1.97 & 10.89 & 1.80 \\
\hline \multirow{3}{*}{$\begin{array}{l}\text { Control } \\
\text { Condition }\end{array}$} & RMSSD & 52.51 & 34.14 & 52.49 & 33.85 \\
\hline & Heart Rate & 70.93 & 11.63 & 71.29 & 11.46 \\
\hline & $\begin{array}{l}\text { Respiratory } \\
\text { Frequency }\end{array}$ & 13.73 & 2.58 & 13.68 & 2.32 \\
\hline
\end{tabular}

Note. RMSSD: Root Mean Square of the Successive Differences 
Slow-paced breathing and executive function

872 Table 2

873 Descriptive statistics for executive functions

\begin{tabular}{lcccc}
\cline { 2 - 5 } & \multicolumn{2}{c}{$\begin{array}{c}\text { Slow-paced breathing } \\
\text { condition }\end{array}$} & \multicolumn{2}{c}{ Control condition } \\
\cline { 2 - 5 } & $M$ & $S D$ & $M$ & $S D$ \\
\hline Stroop interference reaction time (ms) & 143.85 & 68.02 & 138.04 & 72.84 \\
Stroop interference accuracy (number of errors) & 0.32 & 0.47 & 0.58 & 0.73 \\
Automated operation span score & 42.63 & 16.02 & 38.87 & 16.72 \\
Perseverative errors (MCST) & 0.40 & 0.65 & 0.68 & 0.95 \\
\hline
\end{tabular}

Note. MCST: Modified Card Sorting Test 
Slow-paced breathing and executive function

Table $3 \mathrm{a}$ - Correlation between main study variables (Control condition)

\begin{tabular}{|c|c|c|c|c|c|}
\hline & 1 & 2 & 3 & 4 & 5 \\
\hline \multicolumn{6}{|l|}{ 1. Stroop Interference Reaction Time } \\
\hline $\begin{array}{l}\text { 2. Stroop Interference Accuracy (number } \\
\text { of errors) }\end{array}$ & .21 & & & & \\
\hline 3. Automated operation span score & $-.32^{* *}$ & $-.53^{* *}$ & & & \\
\hline 4. Perseverative errors (MCST) & .07 & $.25^{*}$ & -.15 & & \\
\hline $\begin{array}{l}\text { 5. RMSSD (resting measurement before } \\
\text { performing executive tasks) }\end{array}$ & -.11 & $-.30^{* *}$ & $.27^{*}$ & -.20 & \\
\hline \multicolumn{6}{|c|}{ Table $3 \mathrm{~b}$ - Correlation between main study variables (Slow-paced breathing condition) } \\
\hline & 1 & 2 & 3 & 4 & 5 \\
\hline \multicolumn{6}{|l|}{ 1. Stroop Interference Reaction Time } \\
\hline $\begin{array}{l}\text { 2. Stroop Interference Accuracy } \\
\text { (number of errors) }\end{array}$ & .10 & & & & \\
\hline 3. Automated operation span score & -.16 & $-.28^{*}$ & & & \\
\hline 4. Perseverative errors (MCST) & .12 & .21 & $-.34^{* *}$ & & \\
\hline $\begin{array}{l}\text { 5. RMSSD (resting measurement before } \\
\text { performing executive tasks) }\end{array}$ & -.11 & $-.26^{*}$ & $.42^{* *}$ & -.22 & \\
\hline
\end{tabular}

Note: MCST: Modified Card Sorting Test; RMSSD: Root Mean Square of the Successive Differences 